\title{
Leaf open time sinogram (LOTS): a novel approach for patient specific quality assurance of total marrow irradiation
}

\author{
Rajesh Thiyagarajan ${ }^{1,4}$, Dayananda Shamurailatpam Sharma ${ }^{1 *} \mathbb{D}$, Suryakant Kaushik ${ }^{1}$, Mayur Sawant ${ }^{1}$, \\ K. Ganapathy' ${ }^{1}$ N. Arunai Nambi Raj ${ }^{3}$, Srinivas Chilukuri², Sham C. Sundar², Kartikeswar Ch. Patro', \\ Arjunan Manikandan ${ }^{1}$, M. P. Noufal ${ }^{1}$, Rangasamy Sivaraman ${ }^{1}$, Jose Easow ${ }^{5}$ and Rakesh Jalali ${ }^{2}$
}

\begin{abstract}
There is no ideal detector-phantom combination to perform patient specific quality assurance (PSQA) for Total Marrow (TMI) and Lymphoid (TMLI) Irradiation plan. In this study, 3D dose reconstruction using mega voltage computed tomography detectors measured Leaf Open Time Sinogram (LOTS) was investigated for PSQA of TMI/TMLI patients in helical tomotherapy. The feasibility of this method was first validated for ten non-TMI/TMLI patients, by comparing reconstructed dose with (a) ion-chamber (IC) and helical detector array (ArcCheck) measurement and (b) planned dose distribution using 3Dy analysis for 3\%@3mm and dose to $98 \%\left(\mathrm{D}_{98 \%}\right)$ and $2 \%\left(\mathrm{D}_{2 \%}\right)$ of PTVs. Same comparison was extended for ten treatment plans from five TMI/TMLI patients. In all non-TMI/TMLI patients, reconstructed absolute dose was within $\pm 1.80 \%$ of planned and IC measurement. The planned dose distribution agreed with reconstructed and ArcCheck measured dose with mean (SD) 3Dy of 98.70\% (1.57\%) and 2Dy of 99.48\% (0.81\%). The deviation in $\mathrm{D}_{98 \%}$ and $\mathrm{D}_{2 \%}$ were within $1.71 \%$ and $4.10 \%$ respectively. In all 25 measurement locations from TMI/ TMLI patients, planned and IC measured absolute dose agreed within $\pm 1.20 \%$. Although sectorial fluence verification using ArcCHECK measurement for PTVs chest from the five upper body TMI/TMLI plans showed mean \pm SD 2DY of $97.82 \% \pm 1.27 \%$, the reconstruction method resulted poor mean (SD) 3Dy of $92.00 \%( \pm 5.83 \%), 64.80 \%( \pm 28.28 \%$ ), $69.20 \%( \pm 30.46 \%), 60.80 \%$ ( $\pm 19.37 \%)$ and $73.2 \%( \pm 20.36 \%)$ for PTVs brain, chest, torso, limb and upper body respectively. The corresponding deviation in median $D_{98 \%}$ and $D_{2 \%}$ of all PTVs were $<3.80 \%$ and $9.50 \%$. Re-optimization of all upper body TMI/TMLI plans with new pitch and modulation factor of 0.3 and 3 leads significant improvement with 3 Dy of $100 \%$ for all PTVs and median $\mathrm{D}_{98 \%}$ and $\mathrm{D}_{2 \%}<1.6 \%$. LOTS based PSQA for TMI/TMLI is accurate, robust and efficient. A field width, pitch and modulation factor of $5 \mathrm{~cm}, 0.3$ and 3 for upper body TMI/TMLI plan is suggested for better dosimetric outcome and PSQA results.
\end{abstract}

Keywords: Sinogram, Exit dosimetry, Dose Reconstruction, Patient specific QA, Total marrow irradiation, Helical tomotherapy, MVCT

*Correspondence: dayananda.shamurailatpam@gmail.com;

drdayananda_s@apollohospitals.com

${ }^{1}$ Department of Medical Physics, Apollo Proton Cancer Centre, 100 Feet Road Tharamani, Chennai 600096, Tamil Nadu, India

Full list of author information is available at the end of the article

\section{Introduction}

Total body irradiation (TBI) is integral to myeloablative conditioning (MAC) regimen in patients requiring allogeneic bone marrow transplant (ABMT) for myeloid and lymphoid leukemia. The conditioning regimens incorporating TBI have shown better clinical outcome compared to chemotherapy alone [1-3]. However, 
the practice of TBI has witnessed a steady decline as a result of radiation induced toxicity and alternative use of only chemotherapy based conditioning regimens. Profoundly, several investigators have reported the feasibility of selective irradiation of Total Marrow (TMI) and Lymphatic (TMLI), using volumetric modulated arc therapy (VMAT) and more conveniently with helical tomotherapy (HT) [4-7]. TMI have shown a significant reduction of dose to organs at risk (OAR) as compared to TBI, thereby reducing radiation induced toxicity $[5,6,8$, 9] with encouraging complete response rate [9].

The treatment planning of TMI/TMLI using either HT or VMAT is complicated due to extensive and complex irregular shape target volume requiring highly modulated beam intensity, multiple isocenters and field junctions. HT has been the most preferred high precision treatment modality for TMI/TMLI owing to its ability to treat a maximum length of $135 \mathrm{~cm}$ at a time, requiring zero or one junction to treat the entire target volume as compared to 4-5 junctions in VMAT [4-7]. Pretreatment verification of dose delivery in the entire target volume is of paramount importance to ensure accurate delivery of dose to the patient. Conversely, verification of TMI/TMLI treatment plan poses many challenges due to the non-availability of suitable detectors or measuring equipment. All the commercially available active or passive detectors for patient specific quality assurance (PSQA) have limited longitudinal and lateral dimensions of $20-25 \mathrm{~cm}$ to verify highly modulated mega treatment fields from TMI/TMLI especially for HT delivery. The feasibility of different detector arrays (2D or 3D) arranged in planar, hexagonal, circular and helical have been investigated for section by section dose verification of TBI/TMI plans [7, 10, 11]. Takahashi and Hui [12] developed a simple in-house whole body phantom to verify TMI treatment plan of up to $110 \mathrm{~cm}$ in HT using three ionization chambers and three radiochromic films. However, all the investigated detectors and methodologies were complex, unable to measure complete fluence in a single set-up and lack in efficiency.

The unique design of megavoltage cone beam computed tomography (MVCT) detectors in HT allows measurement of delivered sinogram (dose fluence) prior to or during treatment. Several studies have reported the feasibility of using measured sinogram to reconstruct $3 \mathrm{D}$ dose distribution on to the patient CT datasets using in-build or independent dose reconstruction algorithm in routine clinical cases [13-19]. To the best of our knowledge, no study has been conducted to provide an accurate and yet efficient PSQA of TBI/TMI/ TMLI patients treated on HT. In this study, a 3D dose reconstruction method from $\mathrm{MVCT}$ detectors measured leaf open time sinogram (LOTS) was validated for PSQA in a routine clinical environment. The same PSQA method was investigated to assess the delivery accuracy of TMI/TMLI treatment plans. The sensitivity of this PSQA method on HT planning parameter was also investigated for TMI/TMLI patients.

\section{Materials and methods}

\section{Treatment unit}

Radixact X9 used in this study is the latest generation MVCT image guided HT system supplied by Accuray, Inc., Sunnyvale, USA. Although system configuration remains almost the same as its predecessor, the gantry has been redesign to incorporate $\mathrm{kV} \mathrm{X}$-ray based imaging and real-time motion management functionality. However, our model was installed prior to the commercial release of synchrony motion management functionality. The dose rate from the $6 \mathrm{MV}$ flattening filter free (FFF) beam has increased to $1000 \mathrm{MU} / \mathrm{min}$. Intensity modulation is still achieved using 64 binary multi-leaf collimator (MLC), each of $6.25 \mathrm{~mm}$ projected leaf width at isocenter. The user selectable field width along the longitudinal direction remains at $1,2.5$ and $5 \mathrm{~cm}$, whereas maximum lateral dimension is $40 \mathrm{~cm}$. However, the jaws positions along longitudinal direction can be optimize dynamically to achieve better dose conformity in superior and inferior end of the target. A couch catcher assembly installed on the rear side of the gantry supports couch during treatment and reduces the couch sag. The gantry rotation periods remains between 1 to 5 rotation per minute (RPM).

\section{MVCT detector}

The MVCT detector in the Radixact HT system is an arcshaped detector located opposite to the accelerator on the ring gantry. It consists of an array of 640 channels, each with two parallel plate ionization cavities filled with pressurized Xenon gas under 5 atmospheric pressure. The dimension of each detector channel is $1.24 \mathrm{~mm}$ in the transverse and $25.4 \mathrm{~mm}$ in longitudinal direction. The source to detector distance is $145 \mathrm{~cm}$. The imaging field of view (FOV) defined by the width of the MLC is $39.4 \mathrm{~cm}$ at isocenter. Therefore, of the 640 detector channels, 576 are connected to the data acquisition system and only 520 are used in MVCT.

\section{D dose reconstruction from MLC-LOTS}

A unique feature of Radixact HT and Delivery Analysis software (v1.2.2.7, Accuray, Inc., Sunnyvale, USA) is its ability to analyze the exit/transmitted radiation fluence recorded by the in-build MVCT detectors. During the irradiation of a treatment plan in the absence of any object on the TomoCouch, the detector sinogram plot was constructed from the signal pulses collected by 
every three central detector channels corresponding to each MLC leaf. These signal pulses were segmented into projection bins using treatment plan information. In each projection, the pulse value above the set threshold was used for the calculation of LOT. The MLC-LOTS recorded and reconstructed after the end of each irradiation were retrospectively used to reconstruct the dose distribution on the same patient CT dataset in the Delivery Analysis workstation. Readers may refer publications by Kapatoes et al. [13, 14] for detail theoretical explanation and proof of concept.

\section{TMI/TMLI treatment planning}

Treatment plans ( 3 TMI and 2 TMLI) of five adult patients treated during April 2019 till Feb 2020 were used for this retrospective study. Briefly, for each patient, HT treatment plan was generated in Precision (V 2.1.1.1, Accuray, Inc., Sunnyvale, USA) treatment planning system (TPS), separately for the upper and lower body. Planning parameters, shown in Table 1 , were chosen as a balance between plan quality, delivery efficiency and also in alignment with previous publications $[4,7,8]$. Every plan was optimized to deliver a homogeneous dose of $12 \mathrm{~Gy}$ in 6 fractions to the entire target volume. The dose heterogeneity in the junction region of the upper and lower body was maintained within $\pm 5 \%$ of the prescription dose. The optimization engine of the TPS iteratively modify the leaf open time (LOT) of the MLC from different projection angle to closely achieve the defined planning clinical goals. The final dose calculation eliminates LOT less than $20 \mathrm{~ms}$ to minimize the leaf latency related dosimetric error [20]. The resultant LOT of different leaves at different gantry positions were represented as planned sinogram. The outcome of every HT treatment plan were evaluated thoroughly using standard dose volume indices for the targets and OARs and fulfill our pre-defined clinical goals. The detail report on treatment simulation and planning is beyond the scope of this study.

\section{Validation of 3D dose reconstruction and analysis}

The feasibility and accuracy of Delivery Analysis reconstructed 3D dose distribution were first validated for ten non-TMI patients, representing a wide spectrum of clinical sites and level of complexities in treatment plan parameters such as field width, modulation factor and pitch as summarized in Table 1. For each clinically approved treatment plan, pre-treatment PSQA was

Table 1 Helical tomotherapy treatment plan parameters of Non-TMI, TMI and TMLI patients

\begin{tabular}{|c|c|c|c|c|}
\hline \multirow[t]{2}{*}{ Patient } & \multirow[t]{2}{*}{ Diagnosis } & \multicolumn{3}{|c|}{ Treatment plan parameter } \\
\hline & & Field width $(\mathrm{cm})$ & Pitch & $\begin{array}{l}\text { Modulation } \\
\text { factor }\end{array}$ \\
\hline \multicolumn{5}{|c|}{ Non-TMl patients } \\
\hline P1 & Anaplastic oligodendroglioma & 2.5 & 0.43 & 2.20 \\
\hline P2 & Glioblastoma & 1.0 & 0.43 & 2.00 \\
\hline P3 & Glioblastoma & 1.0 & 0.41 & 2.20 \\
\hline P4 & Ca head of pancreas & 1.0 & 0.41 & 2.18 \\
\hline P5 & Oligometastatic Ca Lung & 1.0 & 0.43 & 2.35 \\
\hline P6 & Ca tongue recurrent & 1.0 & 0.41 & 2.35 \\
\hline P7 & Ca right breast & 2.5 & 0.28 & 2.40 \\
\hline P8 & Ca rectum post op & 2.5 & 0.43 & 2.00 \\
\hline P9 & Ca esophagus & 2.5 & 0.30 & 2.10 \\
\hline P10 & Meningioma & 1.0 & 0.41 & 1.90 \\
\hline \multicolumn{5}{|c|}{ TMI/TMLI patients } \\
\hline P11-HFS & Chronic myloid leukemia & 5.0 & 0.31 & 3.50 \\
\hline P11-FFS & & 5.0 & 0.40 & 2.50 \\
\hline P12-HFS & Acute lymphoblastic leukemia & 5.0 & 0.41 & 2.80 \\
\hline P12-FFS & & 5.0 & 0.43 & 2.00 \\
\hline P13-HFS & Acute lymphoblastic leukemia & 5.0 & 0.31 & 3.50 \\
\hline TP13-FFS & & 5.0 & 0.41 & 2.40 \\
\hline P14-HFS & Chronic myloid leukemia & 5.0 & 0.30 & 3.00 \\
\hline P14-FFS & & 5.0 & 0.40 & 2.40 \\
\hline P15-HFS & Acute lymphoblastic leukemia & 5.0 & 0.43 & 2.49 \\
\hline P15-FFS & & 5.0 & 0.40 & 2.15 \\
\hline
\end{tabular}


carried out following departmental protocol of absolute point dose measurement in Cheese phantom (Accuray, Inc., Sunnyvale, USA) using calibrated 0.053 cc Extradin A1SL (Standard Imaging, Inc. Middleton, WI) ionization chamber (IC) and 2D dose fluence measurement using ArcCHECK (SunNuclear, Suntree Blvd Melbourne) helical diode arrays. ArcCHECK is a cylindrical PMMA phantom of $26.6 \mathrm{~cm}$ diameter having $1386\left(0.016 \mathrm{~mm}^{3}\right)$ diodes arranged in a helical manner, providing detector spacing of $10 \mathrm{~mm}$ and covering a treatment length (cranio-caudal) of $20 \mathrm{~cm}$. The measured fluence at the detector plane was unwrapped using ArcCHECK software and compared against the Precision TPS calculated fluence using 2D gamma $(\gamma)$ analysis set at 3\% dose difference at $3 \mathrm{~mm}$ distance-to-agreement (3\%@3mm). The PSQA results were considered acceptable if $95 \%$ of the total number of analyzed pixels have $\gamma$ value less than one.

In addition, the same plans were delivered on Radixact HT without any object on the carbon-fiber flat Tomo couch. The MVCT detector measured LOTS was used to reconstruct the $3 \mathrm{D}$ dose distribution on to the $\mathrm{CT}$ datasets of the patient in Delivery Analysis for verification of absolute point dose and 3D dose distribution either by using 3D gamma analysis or standard dose-volumehistogram (DVH). The same gamma acceptance criteria of 3\%@3mm were used for 3Dy analysis. Whereas, dose to $98 \%\left(D_{98 \%}\right)$ and $2 \%\left(D_{2 \%}\right)$ of planning target volume (PTV) extracted from the cumulative DVHs of planned and reconstructed dose distribution were compared to evaluate agreement in minimum and maximum dose to target.

The potential application of the MLC-LOTS for PSQA was extended for the ten treatment plans (two per patient) of the five previously treated TMI and TMLI patients. For every patient, PSQA plans were created for each of the two clinically approved treatment plans (one each for upper and lower body) by recalculating the dose distribution on a standard cheese phantom. This was required to enable the clinically approved treatment plan to be delivered on Radixact HT in QA mode. However, these plans were delivered on Radixact HT without any phantom. The MVCT detector recorded transmitted radiation from the carbon-fiber Tomo couch were used to reconstruct the MLC-LOTS, which subsequently was used to reconstruct the $3 \mathrm{D}$ dose distribution on the $\mathrm{CT}$ datasets of the patient. Accuracy of the delivered dose fluence was verified by comparing (a) precision TPS calculated (planned) versus IC measured absolute dose in Cheese phantom, (b) planned versus reconstructed 3D dose distribution from measured LOTS using 3D $\gamma$ analysis and (c) planned versus reconstructed 3D dose distribution from measured LOTS using standard dose volume histogram (DVH) of $\mathrm{D}_{98 \%}$ and $\mathrm{D}_{2 \%}$. Based on the verification results, all the five upper body TMI/ TMLI plans were re-optimized using fixed field width of $5 \mathrm{~cm}$, pitch of 0.43 and modulation factor same as in the original plans. To further investigate, the influence of HT planning parameter on the LOTS based PSQA results, another five plans of the upper body TMI/TMLI were created with a field width of $5 \mathrm{~cm}$ and pitch of 0.3 and modulation factor of 3 . All the ten new plans were optimized such that the final dose distribution were comparable or better than the clinically delivered plans. For the ten new upper body TMI/TMLI plans, PSQA based on 3D dose reconstruction from MVCT measured MLC-LOTS were carried out as described above.

\section{Results}

The comparison of planned, measured and reconstructed absolute point dose, $2 \mathrm{D} \gamma$ and $3 \mathrm{D} \gamma, \mathrm{D}_{98 \%}$ and $\mathrm{D}_{2 \%}$ of PTVs of ten non-TMI patients are presented in Table 2. Off the ten clinical plans, planned and ion chamber measured absolute dose agrees within $\pm 1 \%$ in eight and $1.80 \%$ in two plans with overall mean (SD) of $0.1 \%$ $(0.89 \%)$. Whereas, the agreement between reconstructed and IC measured absolute dose was within $\pm 1 \%$ in six and $\pm 1.8 \%$ in four plans, resulting in overall mean (SD) of $0.14 \%(1.03 \%)$. In eight patients, planned dose agrees within $\pm 1 \%$ with that of reconstructed dose while in two patients the agreement was within $\pm 1.2 \%$. In all the plans, both 2Dy and 3Dy were above $96 \%$ with an overall mean (SD) of $99.48 \%$ (0.81\%) for planar dose comparison between planned and ArcCHECK measurement; and $98.7 \%$ (1.57\%) for volumetric dose comparison between planned and reconstructed dose distribution respectively. The planned and reconstructed $\mathrm{D}_{98 \%}$ and $\mathrm{D}_{2 \%}$ to PTVs for the ten non-TMI patients agreed with maximum (mean $\pm \mathrm{SD})$ of $1.71 \%(0.47 \% \pm 0.9 \%)$ and $4.1 \%$ $(1.42 \% \pm 1.39 \%)$ respectively.

The composite dose distribution of upper and lower body treatment plan for a representative TMI patient is shown in Fig. 1. It showed selective irradiation of marrow with the prescribed dose uniformly while sparing the surrounding normal tissues including the island OARs such as lungs, bowels, prostates, etc. The detail analysis of dosimetric outcome is beyond the scope of this study. The comparison of planned and ion chamber measured absolute doses at five representative locations of each TMI/TMLI plans are summarized in Table 3. In all 25 locations, planned and IC measured absolute dose agreed within $\pm 1.2 \%$ ( mean $=0.04 \%, S D=0.55 \%$ ). However, large disagreement was observed between planned and reconstructed 3D dose distribution with many voxels having dose difference more than $3 \%$ at $3 \mathrm{~mm}$ in upper body plan as represented by isogamma 
Table 2 Comparision of absolute point dose, 2D and 3D gamma, absolute dose volume ( $D_{99 \%}$ and $D_{2 \%}$ to PTV) amongst TPS calculated (planned), ion chamber measured and reconstructed dose distribution from LOTS for ten nonTMI patients treated for various clinical sites on RadiXact HT

\begin{tabular}{|c|c|c|c|c|c|c|c|}
\hline \multirow[t]{2}{*}{$\begin{array}{l}\text { Non-TMI } \\
\text { Patient }\end{array}$} & \multicolumn{3}{|c|}{ Absolute dose (cGy) on cheese phantom } & \multicolumn{2}{|c|}{$\begin{array}{l}\text { 2D/3D gamma ( } \gamma \%) \text { values } \\
\text { between TPS calculated and }\end{array}$} & \multicolumn{2}{|c|}{$\begin{array}{l}\text { Deviation (\%) } \\
\text { between reconstructed } \\
\text { and TPS calculated dose }\end{array}$} \\
\hline & Planned & $\begin{array}{l}\text { lon chamber } \\
\text { measured }\end{array}$ & $\begin{array}{l}\text { Reconstructed } \\
\text { from LOTS }\end{array}$ & $\begin{array}{l}\text { ArcCheck } \\
\text { measured } \\
2 \mathrm{D} \gamma \%\end{array}$ & $\begin{array}{l}\text { Reconstructed } \\
\text { from LOTS } \\
3 \mathrm{D} \% \%\end{array}$ & $\mathrm{D}_{98 \%}$ of PTV & $D_{2 \%}$ of PTV \\
\hline P1 & 139 & 137.84 & 140 & 99.4 & 97 & 0.56 & 3.01 \\
\hline P2 & 135 & 134.88 & 135 & 99.8 & 100 & 0.11 & 0.96 \\
\hline P3 & 141 & 140.2 & 141 & 100 & 97 & 1.71 & 4.10 \\
\hline P4 & 172 & 171.51 & 171 & 100 & 100 & -0.31 & -0.15 \\
\hline P5 & 167 & 166.67 & 166 & 99.9 & 100 & -0.61 & -0.49 \\
\hline P6 & 144 & 143.15 & 145 & 100 & 96 & 0.28 & 1.47 \\
\hline P7 & 198 & 201.12 & 200 & 99.5 & 98 & 1.65 & 2.03 \\
\hline P8 & 176 & 175.72 & 178 & 100 & 99 & -0.26 & 1.63 \\
\hline P9 & 149 & 149.65 & 149 & 98.7 & 100 & -0.09 & 1.09 \\
\hline P10 & 122 & 124.13 & 122 & 97.5 & 100 & 1.70 & 0.57 \\
\hline
\end{tabular}

levels greater than one in Fig. 2a for a representative patient. It was also evident from the corresponding $3 \mathrm{Dy}$ plots (Fig. 2b) and comparative DVHs (Fig. 2c) of various PTVs, that planned and reconstructed 3D dose distribution does not agree well both in terms of 3Dy and values of $D_{98 \%}$ and $D_{2 \%}$. Table 4 represents the $3 D \gamma$ of various PTVs resulted from the comparison of planned and reconstructed dose distribution of the five TMI/ TMLI patients. None of the upper body plans pass 3Dy criteria of $95 \%$ to all PTVs, although it was comparatively better for patients P13 and P14. The mean (SD) 3Dy for PTVs brain, chest, torso, limb, combination of all PTVs in upper body, external of upper body and lower body were $92.00 \%$ (5.83\%), 64.80\% (28.28\%), 69.20\% (30.46\%), $60.80 \%(19.37 \%), 73.2 \%(20.36 \%) 84.00 \%(11.98 \%)$ and $100 \%(0 \%)$ respectively. However, all the lower body plans showed excellent agreement with mean 3Dy of $100 \%$ for PTV lower body and external body. Figure 3a shows the deviation between planned and reconstructed $D_{98 \%}$ and $\mathrm{D}_{2 \%}$ of various PTVs. The median deviation in $\mathrm{D}_{98 \%}$ of various PTVs were $-0.60,2.30,3.79,1.78,1.20$ and 1.39 for brain, chest, torso, limb, upper body and lower body respectively. The corresponding deviations in $\mathrm{D}_{2 \%}$ were larger in all PTVs with 2.22, 5.56, 6.23, 4.68, 4.09 and 9.48 respectively.

As a part of the TMI/TMLI implementation protocol in our Institute, pre-treatment planar dose verification of every patient was carried out only for chest PTVs by comparing planned and ArcCHECK measured dose distribution. All patients showed 2Dy (3\%@3mm) above $96 \%$ with a mean \pm SD of $97.82 \% \pm 1.27 \%$ as compared to $3 \mathrm{D} \gamma$ of $64.80 \% \pm 28.28 \%$ from reconstruction method. The poor disagreement between $2 \mathrm{D} \gamma$ and $3 \mathrm{D} \gamma$ of the upper body TMI/TMLI plans lead us to investigate the influence of HT plan parameters on the 3Dy passing rate. First, all the five upper body original TMI/TMLI plans were re-optimize by changing the pitch to 0.43 while retaining the original modulation factor (2.49 to 3.5$)$ and field width. Secondly, all the five original upper body TMI/TMLI plans were re-optimized using fix field width of $5 \mathrm{~cm}$, pitch and modulation factor of 0.3 and 3 respectively. The ten new plans thus created were dosimetrically comparable or slightly better as compare to clinically delivered (original) plans. Analysis of MLC-LOTS of clinically delivered plans and new plans with pitch of 0.3 and modulation factor of 3 lead to the reduction of maximum LOT from 15.06 to $21.41 \%$ in four of five plans with reduced standard deviation (SD) ranging from 0.73 to $27.26 \%$ in all plans. The mean LOT were also reduced ranging from 9.82 to $31.29 \%$ in three patients while it was increase by around 3\% in two plans. The average reduction of maximum and mean LOT for the five new plans were $14.95 \%$ and $14.86 \%$ respectively. In contrast, the change of pitch to 0.43 without changing the modulation factor and field width, lead to an overall increase in the average value of maximum and mean LOT to $29.48 \%$ and $18.25 \%$ respectively. All the ten newly generated plans were exposed to Radixact HT as described before and measured LOTS were used to reconstruct the 3D dose distribution on to the same patient $\mathrm{CT}$ datasets. In all five new upper body plans with new pitch of $0.43,3 \mathrm{Dy}$ (3\%@3mm) were less than the acceptable criteria of 95\% 


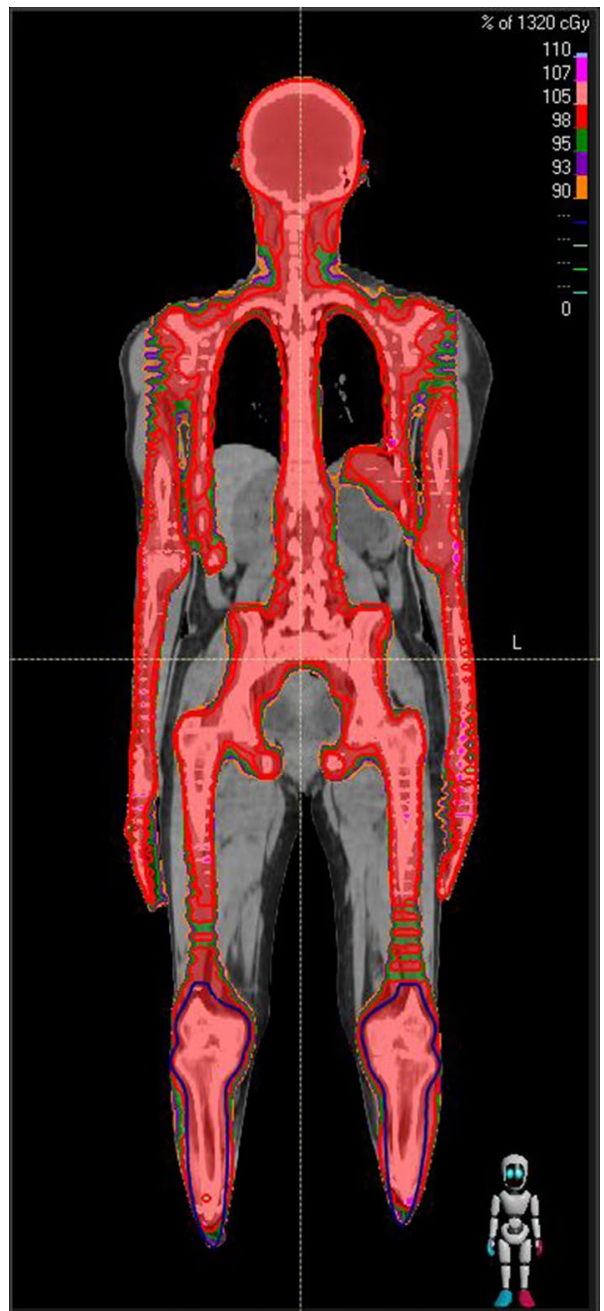

Fig. 1 Composite dose distribution from upper and lower body plans of a representative TMI patients showing selective irradiation of total marrow

for majority of the PTVs. Correspondingly, the agreement of planned and reconstructed values of $\mathrm{D}_{98 \%}$ and $\mathrm{D}_{2 \%}$ showed large deviation as shown in Fig. 3b. However, for the other five new plans with pitch of 0.3 and modulation factor of 3 , planned and reconstructed dose agrees with 3Dy (3\%@3mm) values of $100 \%$ for all PTVs and external bodies. The agreement even at $2 \% @ 2 \mathrm{~mm}$ criteria were $\geq 94 \%$ in all plans and for all PTVs with mean (SD) of $97.6 \%(2.51 \%)$, 98.6\% (2.61\%), 99.6\% (0.89\%), 98.4\% (2.3\%), 98.4\% (1.52\%) and 99.6\% (0.55\%) for brain, chest, torso, limb, upper body and external respectively (Table 4). This also leads to significant improvement in the agreement of planned and reconstructed values of $\mathrm{D}_{98 \%}(p=0.001)$ and $\mathrm{D}_{2 \%}(p=0.001)$ for all PTVs in all plans (Fig. 3c). The median deviation in $\mathrm{D}_{98 \%}\left(\mathrm{D}_{2 \%}\right)$ of PTVs brain, chest, torso, limb and upper body were $-1.52 \%(-1.37 \%),-0.98 \%(-0.42 \%),-1.40 \%(-1.21 \%)$, $-1.30 \%(-0.92 \%),-1.13 \%(-1.07 \%)$ respectively.

\section{Discussion}

Our current standard protocol for pre-treatment PSQA of TMI/TMLI patient treatment on Radixact HT include verification of absolute dose in five locations corresponding to brain, chest, pelvis, upper leg and lower leg using calibrated ionization chamber inserted in Cheese phantom and 2D fluence verification only for the chest target using ArcCHECK helical detector array. ArcCHECK allows measurement of treatment field length $\leq 20 \mathrm{~cm}$ in a single irradiation. Our protocol is in alignment with other studies wherein several authors have reported verification of absolute dose and fluence either section by section or in the junction of two consecutive arcs using various detectors and methods $[7,11]$. In addition, for each patient we also carried out in-vivo EBT3 film dosimetry (a) at eleven pre-defined locations across the whole body and (b) in the junction region of upper and lower body treatment plans to ensure delivery of homogeneous dose. The excellent agreement between planned and measured absolute dose $(< \pm 1.2 \%)$ and planar dose fluence $(2 \mathrm{D} \gamma>96 \%)$ in the five TMI/TMLI patients were within the internationally acceptable criteria. Off the many sub PTVs, we have limited the fluence verification only for the PTV chest due to logical and technical challenges prior to treatment and postulated that the results may be still applicable to other PTVs. We have chosen PTV chest because of its complicated shape with many OARs located as an island and hence represent the most complex intensity fluence. The probable shortcoming of our protocol is extensive time spend for the delivery QA, verification of limited dose fluence of a mega treatment volume, challenges in the in-vivo absolute dose verification in a highly modulated heterogeneous dose region and non-availability of on-line or prior-treatment in-vivo verification results. Although the PSQA method reported by Takahashi [12] was carried out in a single irradiation, it requires a customized phantom with many slabs, three ion chambers and three films stitched one after the other to cover $100 \mathrm{~cm}$ length of the target. Besides extensive logistic requirements, it is very labor intensive, time consuming and co-registration of film measured and TPS calculated dose fluence is not straight forward and error prone.

The feasibility of using MVCT measured LOTS to reconstruct $3 \mathrm{D}$ dose distribution on to patient $\mathrm{CT}$ datasets have been reported by several authors either by using in-build or independent dose reconstruction algorithm in routine clinical cases [13-19, 21]. The 


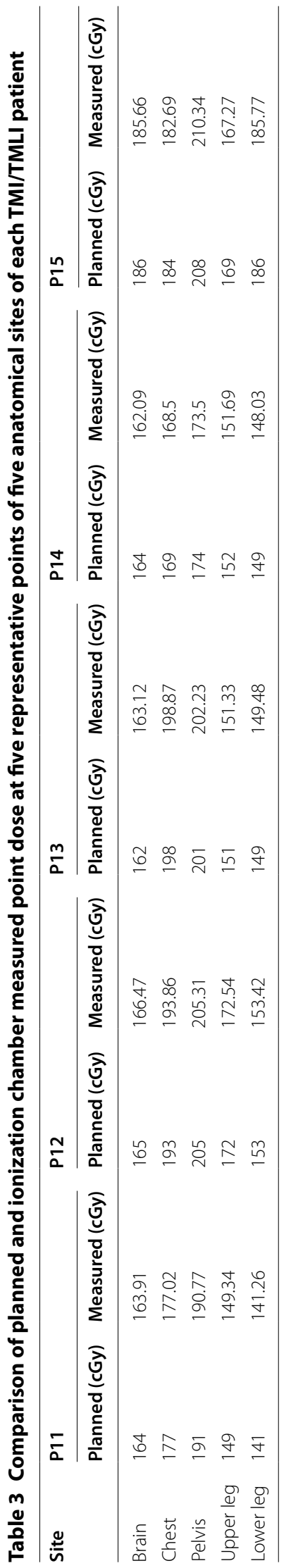



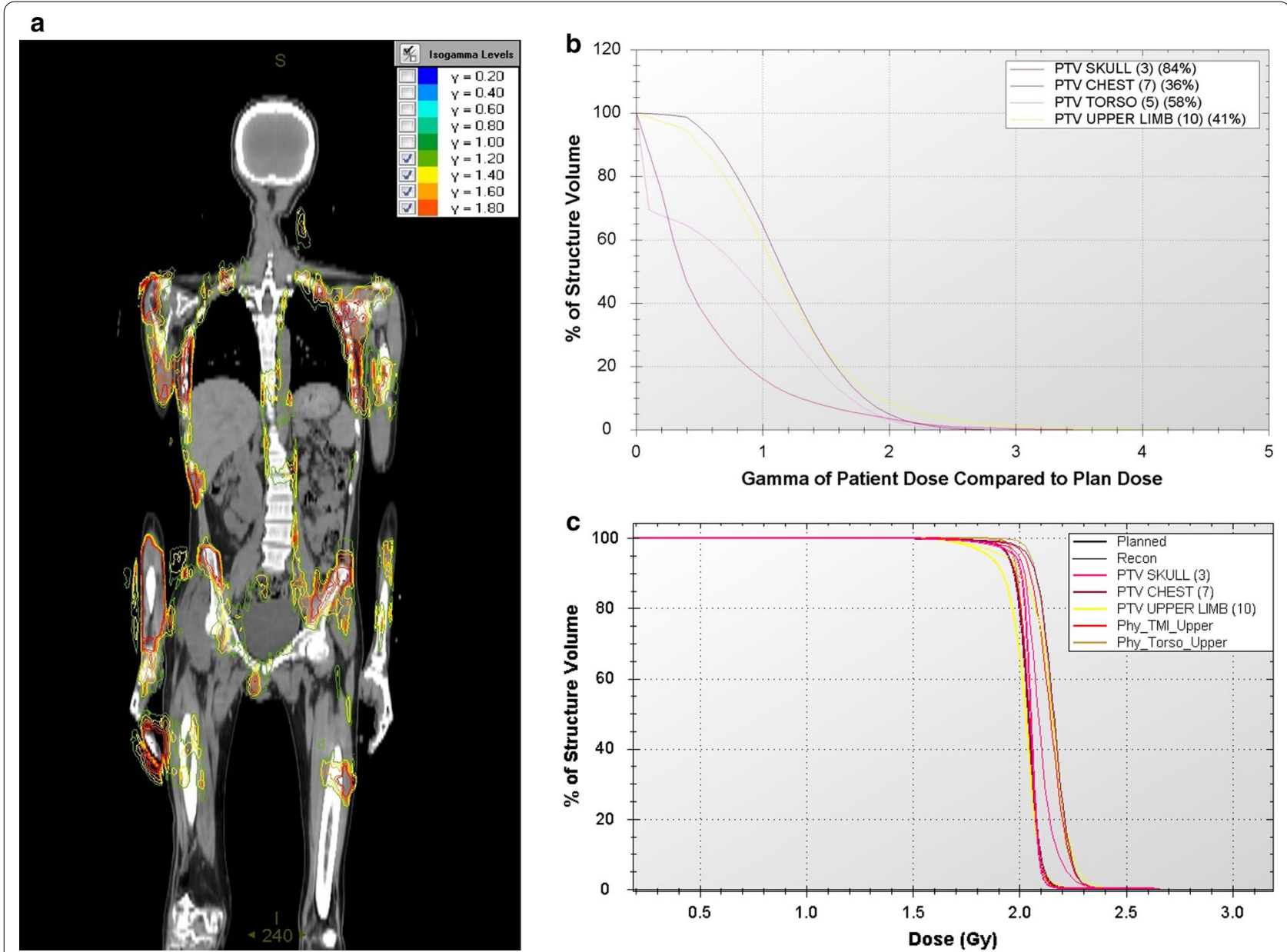

Fig. 2 a lsogamma levels greater than one resulted from the comparison of planned and reconstructed dose distribution from measured LOTS for one of the representative upper body TMI treatment plan. 3D y was analyse using $3 \%$ dose difference at $3 \mathrm{~mm}$ distance to agreement. $\mathbf{b}$ 3D p plots of four separate planning target volumes (PTVs) in upper body TMI plan of a representative patient. c Comparison of planned and reconstructed cumulative dose volume histogram (DVH) of four separate planning target volumes (PTVs) in upper body TMI plan

excellent agreement both in absolute dose $(< \pm 1.8 \%)$, $2 \mathrm{D \gamma}(>97 \%), 3 \mathrm{D} \gamma(>96 \%)$ and $2 \mathrm{D \gamma}$ vs $3 \mathrm{D} \gamma$ variation $(\leq 2.5 \%)$ between reconstructed dose from LOTS as compared to planned, ion chamber and ArcCHECK measurement, in all ten non-TMI patients validated the accuracy and reliability of LOTS reconstructed method in Delivery Analysis against the standard methods. Our results are in agreement with previous publications [1719]. Although MVCT measured LOTS reconstructed 3D dose distribution has been successfully implemented as an alternative PSQA method for regular clinical cases, its feasibility in TMI/TMLI has not been reported so far. The feasibility and validation of this method for TMI/TMLI is especially important as there is no direct approach and suitable detector or phantom to verify the delivery accuracy of this complex and highly modulated mega field.
In a big surprise to our retrospective investigation of LOTS reconstructed 3D dose distribution in five TMI/ TMLI patients, 3Dy to the majority of the PTVs in upper body plans were found much below the acceptable criteria of 95\%. Amongst the four separate PTVs (Brain, Chest, Torso and limb), 3Dy were slightly better for brain (>90\%) except P12. The 3Dy values (28\%-94\%) of PTV Chest of every patient were much lesser than corresponding 2Dy (>96\%) estimated from ArcCHECK measurement. For reasons not clear to us, we observed poor $3 \mathrm{Dy}$ values from the analysis of the first patient (P11) itself. In an attempt to remove any possible error, we carried out a series of investigations including various email communication and data sharing with Accuray Medical Physics support team based in Europe. MVCT detector output was re-calibrated and baselined again following recommended protocol described in the manual. As per the suggestions from Accuray medical physics support, 
Table 4 3D gamma ( $(\%)$ values resulted from the comparison of planned and reconstructed dose distribution in 6 planning target volumes (PTVs) and two external (body) from the two plans (upper body and lower body) of every TMI/ TMLI patients. 3D $\gamma$ values of 3\%@3mm from the original plans were obtained with different pitch and modulation factor listed in Table 1, whereas 3Dy values of $3 \% @ 3 \mathrm{~mm}$ with pich modified to 0.43 from the original plans were also shown here. The 3Dy values with $2 \% @ 2 \mathrm{~mm}$ from another set of new plans of the same patients with fixed pitch of 0.3 and modulation factor of 3 showed significant improvement in the $3 D_{\gamma}$ values

\begin{tabular}{|c|c|c|c|c|c|c|c|c|c|c|c|c|c|c|c|c|}
\hline \multirow[t]{2}{*}{ Treatment plan } & \multirow[t]{2}{*}{ Target (PTV) } & \multicolumn{5}{|c|}{$\begin{array}{l}3 \mathrm{D} \gamma(3 \% @ 3 \mathrm{~mm}) \text { from original } \\
\text { plans }\end{array}$} & \multicolumn{5}{|c|}{$\begin{array}{l}\text { 3D }(3 \% @ 3 \mathrm{~mm}) \text { from new } \\
\text { plans with field width }=5 \mathrm{~cm} ; \\
\text { pitch }=0.43 \text { and modulation } \\
\text { factor = same as original plans }\end{array}$} & \multicolumn{5}{|c|}{$\begin{array}{l}3 \mathrm{D} y(2 \% @ 2 \mathrm{~mm}) \text { from new } \\
\text { plans with field width }=5 \mathrm{~cm} \text {; } \\
\text { pitch }=0.3 \text { and modulation } \\
\text { Factor }=3\end{array}$} \\
\hline & & P11 & P12 & P13 & P14 & P15 & P11 & P12 & P13 & P14 & P15 & P11 & P12 & P13 & P14 & P15 \\
\hline \multirow[t]{6}{*}{ HFS } & Brain & 90 & 83 & 97 & 97 & 93 & 72 & 96 & 95 & 92 & 99 & 100 & 94 & 97 & 97 & 100 \\
\hline & Chest & 75 & 28 & 86 & 94 & 41 & 53 & 57 & 68 & 63 & 60 & 100 & 99 & 94 & 100 & 100 \\
\hline & Torso & 83 & 52 & 94 & 93 & 24 & 60 & 68 & 82 & 51 & 46 & 100 & 98 & 100 & 100 & 100 \\
\hline & Limb & 85 & 34 & 73 & 55 & 57 & 82 & 50 & 55 & 51 & 44 & 100 & 95 & 97 & 100 & 100 \\
\hline & Upper body & 86 & 50 & 91 & 87 & 52 & 67 & 69 & 78 & 63 & 63 & 100 & 97 & 97 & 98 & 100 \\
\hline & External upper body & 94 & 68 & 95 & 88 & 75 & 87 & 79 & 88 & 82 & 77 & 100 & 99 & 99 & 100 & 100 \\
\hline \multirow[t]{2}{*}{ FFS } & Lower body & 100 & 100 & 100 & 100 & 100 & & & & & & & & & & \\
\hline & External lower body & 100 & 100 & 100 & 100 & 100 & & & & & & & & & & \\
\hline
\end{tabular}

the threshold of LOT was decreased from 0.7 to 0.5 . Even after all these probable corrective measures also, the new $3 \mathrm{D}$ dose distribution reconstructed for the same patient (P11) from the newly measured LOTS resulted in no changes in the 3Dy values of all PTVs. Despite this unsatisfactory result, we have continued the measurement of LOTS for the other four patients and reconstructed dose distributions were compared with the corresponding plans. Off the five patients, the upper body TMI/ TMLI plan of P14, which was created using $5 \mathrm{~cm}$ field width, pitch of 0.3 and modulation factor of 3 showed the best agreement with $3 \mathrm{D} \gamma$ values $\geq 93 \%$ in three separate PTVs. Although 3Dy values were very poor for all PTVs and for every patient, the median deviation in $\mathrm{D}_{98 \%}$ of all PTVs were within 2.5\% except torso where the deviation was $3.78 \%$. The deviation in $\mathrm{D}_{2 \%}$ was relatively large for all PTVs and increase up to $9.48 \%$ for lower body PTV where 3Dy were $100 \%$. Overall, minimum PTV coverage $\left(D_{98 \%}\right)$ from the reconstruction method was within $\pm 5 \%$ of corresponding plans except for PTV upper body where a reduction of up to $6.33 \%$ was observed. The reconstruction method increases hot spot $\left(\mathrm{D}_{2 \%}\right)$ to all PTVs by up to $12.51 \%$ as compare to plan. The overall analysis results based on minimum and maximum dose to PTVs can still be considered acceptable, although not very satisfactory, based on the complexity of the target and treatment technique.

The selection of optimum field width, pitch and modulation factor determine both TMI/TMLI plan quality and treatment time. Hui et al. [4] have investigated the effect of field width, modulation factor and pitch on the treatment plan outcome and delivery time. The authors have reported a reduction of treatment delivery time by $50 \%$ when the field width was increased from 2.5 to $5 \mathrm{~cm}$ in superior-inferior direction. For a $5 \mathrm{~cm}$ field width, earlier studies have recommended modulation factor and pitch ranging from 2.0 to 2.8 and 0.397 to $0.46[4,7,8]$ respectively. The impact of HT planning parameters on the PSQA results especially with reconstruction from measured LOTS has not been reported in the literature. In all the lower body TMI/TMLI plans where the $3 \mathrm{D} \gamma$ values were $100 \%$, a field width of $5 \mathrm{~cm}$, modulation factor from 2.15 to 2.5 and pitch from 0.4 to 0.41 were used, which is in agreement with the reported values $[4,7,8]$. However, in the upper body TMI/TMLI plans, modulation factor and pitch were customized from 2.49 to 3.5 and 0.3 to 0.43 to meet the set clinical goals. Although both modulation factor of 2.49 and 3.5 provides a poor $3 \mathrm{D}$ y values in patients $\mathrm{P} 11$ and P15, we observed a fairly better 3Dy values of P14 plan created with modulation factor of 3 and pitch of 0.3 . Subsequently, all the upper body TMI/TMLI plans including for patient P14 were re-optimized with $5 \mathrm{~cm}$ field width, pitch of 0.3 and modulation factor of 3 . Moreover, even after getting optimum dose distribution and MLC-LOT, we continue to run up to 1000 iteration, while simultaneously ensuring no change in the plan quality. The reconstructed dose from the new upper body plans thus created showed improvement both in $3 \mathrm{D} \gamma$ and minimum target coverage and hot spot. As the 

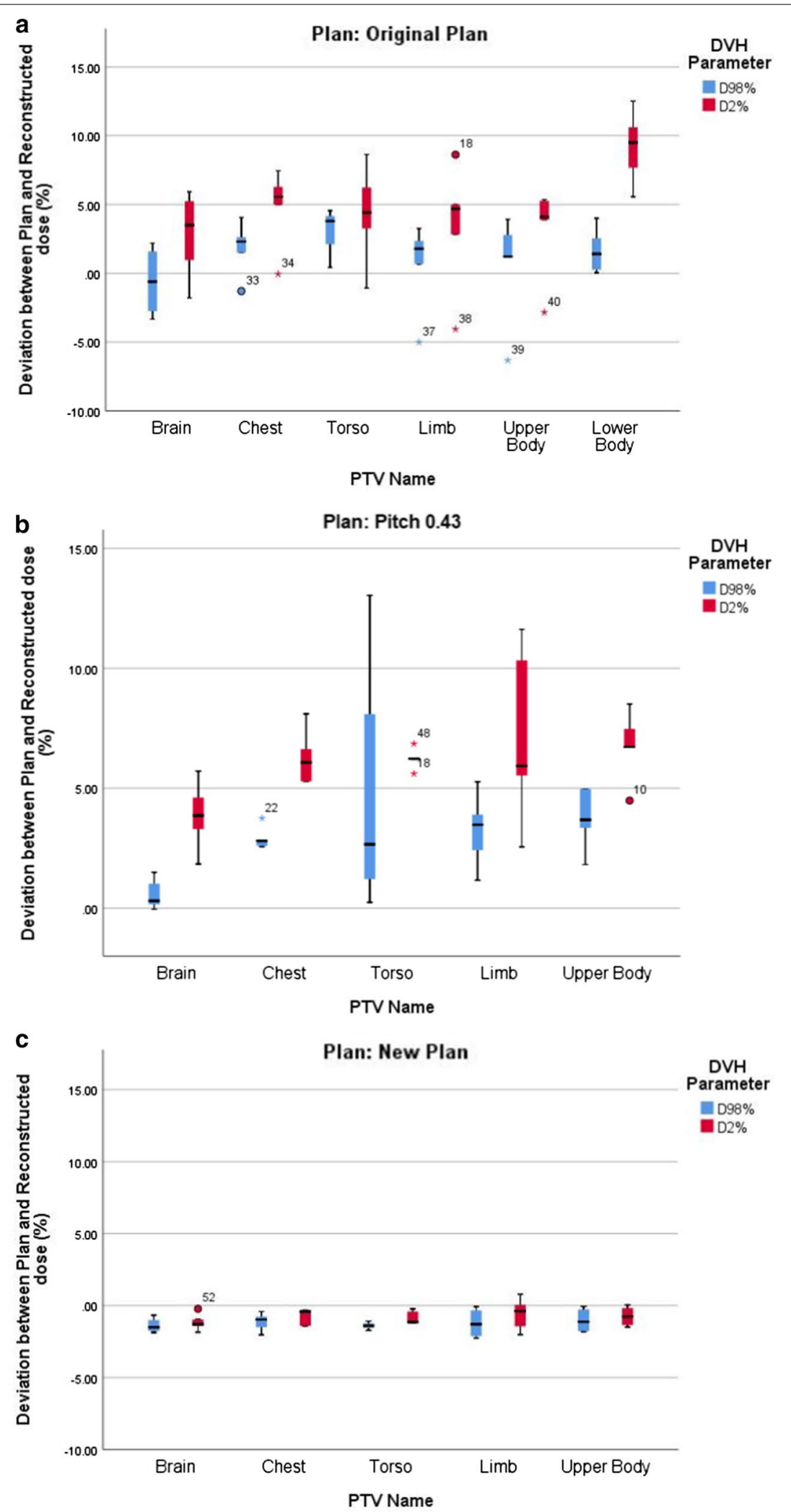

Fig. 3 a Deviation between planned and reconstructed $D_{98 \%}$ and $D_{2 \%}$ of various PTVs from the (a) original TMI/TMLI plans. b new TMI/TMLI plans with new pitch of 0.43 and modulation factor same as original plans. c new TMI/TMLI plans with new pitch of 0.3 and modulation factor of 3 
3 Dy was $100 \%$ for all plans and PTVs, we have tightened the evaluation criteria to $\% @ 2 \mathrm{~mm}$. Even at this stringent evaluation criteria also, almost all plans pass acceptance criteria of $95 \%$. This also leads to improvement in the deviation in $\mathrm{D}_{98 \%}$ and $\mathrm{D}_{2 \%}$. However, only increase of pitch to 0.43 without changing other plan parameters did not resulted any improvement both in $3 \mathrm{D} \gamma, \mathrm{D}_{98 \%}$ and $\mathrm{D}_{2 \%}$.

Although, all the original plans were deliverable on RadiXact HT and passed the traditional ion chamber and ArcCHECK based PSQA, the used HT plan parameters were not optimum for MLC-LOTS based PSQA methods. We proposed a field width of $5 \mathrm{~cm}$, pitch of 0.3 and modulation factor of 3 as an optimum HT plan parameters for TMI/TMLI patients not only to create the most optimum dose distribution but also for a successful implementation of MLC-LOTS based PSQA methods. Measured LOTS based reconstructed methods provide accurate and efficient verification of TMI/TMLI plan in a single irradiation. Reconstructed 3D dose calculation assumes that there is no change in the patient anatomy and tumor geometry. Moreover, the reconstruction method in Delivery Analysis does not explicitly check for differences between planned and delivered gantry angle, couch position, or treatment field position. Only variations in MLC-LOT are considered when calculating dose differences.

\section{Conclusion}

MLC-LOTS based PSQA is accurate, robust and easy to implement in any busy radiation oncology facility without additional logistics, new phantom and detector. It serves as an effective and efficient method of PSQA for HT treatment plans and best suited for very large target like TMI/TMLI. HT treatment plan parameters, mean and maximum LOT greatly influence the reconstructed $3 \mathrm{D} \gamma, \mathrm{D}_{98 \%}$ and $\mathrm{D}_{2 \%}$. We proposed a field width of $5 \mathrm{~cm}$, pitch of 0.3 and modulation factor of 3 as an optimum HT plan parameters for TMI/TMLI patients not only to create the most optimum dose distribution but also for a successful implementation of MLC-LOTS based PSQA methods.

\footnotetext{
Abbreviations

TBI: Total body irradiation; MAC: Myeloablative conditioning; ABMT: Allogeneic bone marrow transplant; TMI: Total marrow irradiation; TMLI:Total marrow and lymphoid irradiation; VMAT: Volumetric modulated arc therapy; HT: Helical tomotherapy; PSQA: Patient specific quality assurance; MVCT: Megavoltage cone beam computed tomography; LOTS: Leaf open time sinogram; TPS: Treatment planning system; FFF: Flattening filter free; MLC: Multi-leaf collimator; IC: Ionization chamber; ү: Gamma; 3\%@3mm: 3\% Dose difference at $3 \mathrm{~mm}$ distance-to-agreement; $\mathrm{DVH}$ : Dose-volume-histogram; $\mathrm{D}_{98 \%}$ : Dose to $98 \%$ volume; $\mathrm{D}_{20}$ : Dose to $2 \%$ volume; PTV: Planning target volume; $\mathrm{SD}$ : Standard deviation.
}

Acknowledgements

We would like to acknowledge Mr Dylan Casey and Mr Bargavan from Accuray for their physics support.

\section{Authors' contributions}

As per the authorship policy. All authors read and approved the final manuscript.

\section{Funding}

Nil.

Availability of data and materials

Yes, will be supply when requested.

\section{Ethical approval and consent to participate}

The study does not contain any direct involvement of patient or animal. All measurements are performed on phantom. As per institution policy, no need for ethical approval.

\section{Consent for publication \\ Agreed.}

\section{Competing interests}

The authors declare that they have no competing interests.

\section{Author details}

${ }^{1}$ Department of Medical Physics, Apollo Proton Cancer Centre, 100 Feet Road Tharamani, Chennai 600096, Tamil Nadu, India. ${ }^{2}$ Department of Radiation Oncology, Apollo Proton Cancer Centre, 100 Feet Road Tharamani, Chennai 600096, Tamil Nadu, India. ${ }^{3}$ Centre for Biomaterials, Cellular and MolecuIar Theranostics (CBCMT), VIT University, Vellore 632014, India. ${ }^{4}$ School of Advanced Sciences, VIT University, Vellore 632014, India. ${ }^{5}$ Department of Haematology, Blood and Marrow Transplantation, Apollo Speciality Hospital, Teynampet, Chennai, India.

Received: 27 April 2020 Accepted: 17 September 2020

Published online: 14 October 2020

\section{References}

1. Ringden O, Ruutu T, Remberger M, Nikoskelainen J, Volin L, Vindelov L, Parkkali T, Lenhoff S, Sallerfors B, Ljungman P. A randomized trial comparing busulfan with total body irradiation as conditioning in allogeneic marrow transplant recipients with leukemia: a report from the Nordic Bone Marrow Transplantation Group. Blood. 1994:83:2723-30.

2. Dusenbery K, Daniels K, McClure J, McGlaver P, Ramsay N, Blazar B, Neglia J, Kersey J, Woods W. Randomized comparison of cyclophosphamide-total body irradiation versus busulfan-cyclophosphamide conditioning in autologous bone marrow transplantation for acute myeloid leukemia. Int J Radiat Oncol Biol Phys. 1995;31(1):119-28.

3. Blaise D, Maraninchi D, Michallet M, Reiffers J, Jouet J, Milpied N, et al. Long-term follow-up of a randomized trial comparing the combination of cyclophosphamide with total body irradiation or busulfan as conditioning regimen for patients receiving HLA-identical marrow grafts for acute myeloblastic leukemia in first complete remission. Blood. 2001;97(11):3669-71.

4. Hui S, Kapatoes J, Fowler J, Henderson D, Olivera G, Manon R, Gerbi B, Mackie T, Welsh J. Feasibility study of helical tomotherapy for total body or total marrow irradiation. Med Phys. 2005;32:3214-24.

5. Aydogan B, Yeginer M, Kavak G, Fan J, Radosevich J, Gwe-Ya K. Total marrow irradiation with rapidarc volumetric arc therapy. Int J Radiat Oncol Biol Phys. 2011:81:592-9.

6. Fogliata A, Cozzi L, Clivio A, Ibatici A, Mancosu P, Navarria P, Nicolini G, Santoro A, Vanetti E, Scorsetti M. Preclinical assessment of volumetric modulated arc therapy for total marrow irradiation. Int J Radiat Oncol Biol Phys. 2011;80:628-36.

7. Haraldsson A, Engellau J, Lenhoff S, Engelholm S, Bäck S, Engström P. Implementing safe and robust Total Marrow Irradiation using Helical Tomotherapy—a practical guide. Phys Med. 2019;60:162-7.

8. Schultheiss T, Wong J, Liu A, Olivera G, Somlo G. Image-guided total marrow and total lymphatic irradiation using helical tomotherapy. Int J Radiat Oncol Biol Phys. 2007;67:1259-67. 
9. Somlo G, Spielberger R, Frankel P, Karanes C, Krishnan A, Parker P, Popplewell L, Sahebi F, Kogut N, Snyder D, et al. Total marrow irradiation: a new ablative regimen as part of tandem autologous stem cell transplantation for patients with multiple myeloma. Clin Cancer Res. 2011;17:174-82.

10. Liang Y, Kim G, Pawlicki T, Mundt A, Mell L. Feasibility study on dosimetry verification of volumetric-modulated arc therapy-based total marrow irradiation. J Appl Clin Med Phys. 2013;14:15-23.

11. Morrison C, Symons K, Woodings S, House M. Verification of junction dose between VMAT arcs of total body irradiation using a Sun Nuclear ArcCHECK phantom. J Appl Clin Med Phys. 2017;18:177-82.

12. Takahashi Y, Hui S. Fast, simple, and informative patient-specific dose verification method for intensity modulated total marrow irradiation with helical tomotherapy. Radiat Oncol. 2014;34:9.

13. Kapatoes J, Olivera G, Reckwerdt P, Fitchard E, Schloesser E, Mackie T. Delivery verification in sequential and helical tomotherapy. Phys Med and Biol. 1999:44:1815-41.

14. Kapatoes J, Olivera G, Ruchala K, Smilowitz J, Reckwerdt P, Mackie T. A feasible method for clinical delivery verification and dose reconstruction in tomotherapy. Med Phys. 2001;28:528-42.

15. Sheng K, Jones R, Yang W, Saraiya S, Schneider B, Chen Q, Sobering G, Olivera G, Read P. 3D dose verification using tomotherapy CT detector array. Int J Radiat Oncol Biol Phys. 2012:82:1013-20.

16. Handsfield L, Jones R, Wilson D, Siebers J, Read P, Chen Q. Phantomless patient-specific TomoTherapy QA via delivery performance monitoring and a secondary Monte Carlo dose calculation. Med Phys. 2014;41(10):1703.
17. Pisaturo O, Miéville F, Tercier P, Allal A. An efficient procedure for tomotherapy treatment plan verification using the on-board detector. Phys Med Biol. 2015;60:1625-39

18. Deshpande S, Xing A, Metcalfe P, Holloway L, Vial P, Geurts M. Clinical implementation of an exit detector-based dose reconstruction tool for helical tomotherapy delivery quality assurance. Med Phys. 2017:44:5457-66.

19. Chung E, Kwon D, Park T, Kang H, Chung Y. Clinical implementation of Dosimetry Check ${ }^{\mathrm{TM}}$ for TomoTherapy ${ }^{\otimes}$ delivery quality assurance. J Appl Clin Med Phys. 2018;19:193-9.

20. Kissick M, Fenwick J, James J, Jeraj R, Kapatoes J, Keller H, Mackie T, Olivera G, Soisson E. The helical tomotherapy thread effect. Med Phys. 2005;32:1414-23

21. Westerly DC, Soisson E, Chen YQ, Woch ZK, Schubert YL, Olivera S, Mackie TR. Treatment planning to improve delivery accuracy and patient throughput in helical tomotherapy. Int J Radiat Oncol Biol Phys. 2009;74(4):1290-7. https ://doi.org/10.1016/j.jijobp.2009.02.004.

\section{Publisher's Note}

Springer Nature remains neutral with regard to jurisdictional claims in published maps and institutional affiliations.
Ready to submit your research? Choose BMC and benefit from:

- fast, convenient online submission

- thorough peer review by experienced researchers in your field

- rapid publication on acceptance

- support for research data, including large and complex data types

- gold Open Access which fosters wider collaboration and increased citations

- maximum visibility for your research: over $100 \mathrm{M}$ website views per year

At $\mathrm{BMC}$, research is always in progress.

Learn more biomedcentral.com/submissions 